Para enlazar con este artículo / To link to this article:

http://dx.doi.org/10.14198/fem.2019.33.10

Para citar este artículo / To cite this article:

Cavalo, Lucía Elena. «Androcentrismo y espacio público: análisis exploratorio sobre la subrepresentación femenina en la nomenclatura urbana de la Ciudad Autónoma de Buenos Aires». En Feminismo/s, 33 (junio 2019): 249-271. DOI: $10.14198 / \mathrm{fem} .2019 .33 .10$

\title{
ANDROCENTRISMO Y ESPACIO PÚBLICO: ANÁLISIS EXPLORATORIO SOBRE LA SUBREPRESENTACIÓN FEMENINA EN LA NOMENCLATURA URBANA DE LA CIUDAD AUTÓNOMA DE BUENOS AIRES
}

\section{ANDROCENTRISM AND PUBLIC SPACE: EXPLORATORY ANALYSIS ON THE UNDER-REPRESENTATION OF WOMEN IN THE URBAN NOMENCLATURE OF THE CITY OF BUENOS AIRES}

\begin{abstract}
Lucía Elena CAVALO
Facultad de Ciencias Sociales, Universidad de Buenos Aires (UBA) - Universidad de Ciencias Empresariales y Sociales (UCES)

lucia.cavalo@hotmail.com orcid.org/0000-0001-5253-1215
\end{abstract}

\section{Resumen}

Con el advenimiento del orden industrial moderno, el espacio público quedó instituido como una esfera de dominio masculino. En concordancia, a lo largo de la historia, las ciudades fueron marcadas material y simbólicamente por aquellos varones que constituyen el centro hegemónico de la vida social y que han asumido el espacio público como propio.

Asumiendo que las ciudades constituyen verdaderos macrotextos susceptibles de ser leídos e interpretados, este estudio ofrece un primer acercamiento a las maneras en que las asimetrías de género persistentes en nuestras sociedades encuentran continuidad en la trama simbólica de las ciudades. Con este propósito, se presenta un análisis cuantitativo de la nomenclatura urbana de la Ciudad Autónoma de Buenos 
Androcentrismo y espacio público: análisis exploratorio sobre la subrepresentación

femenina en la nomenclatura urbana de la Ciudad Autónoma de Buenos Aires

Aires que, asumiendo una perspectiva de género, indaga los modos y grados en que las mujeres encuentran acogida en los nombres de las calles de esta ciudad.

Palabras clave: derechos de la mujer, espacio público, ciudades, patriarcado, equidad de género.

\section{Abstract}

With the advent of the modern industrial order, public space was instituted as a sphere of male domination. Accordingly, through out history, cities were marked materially and symbolically by those men who constitute the hegemonic center of social life and who have assumed public space as their own.

Assuming that cities constitute true macrotexts capable of being read and interpreted, this study offers a first approach to the ways in which the persistent gender asymmetries in our societies find continuity in the symbolic weft of cities. With this purpose, we present a quantitative analysis of the urban nomenclature of the Autonomous City of Buenos Aires that, assuming a gender perspective, investigates the ways and degrees in which women are taken in in the names of the streets of this city.

Keywords: women's rights, public space, cities, patriarchy, gender equality.

\section{INTRODUCCIÓN. LA CIUDAD COMO MACROTEXTO}

«Las normas de género intervienen en todo lo relativo a los modos y grados en que podemos aparecer en el espacio público» Judith Butler

Los estudios sobre las ciudades se han centrado mayormente en aspectos asociados a la dimensión material del espacio público, a pesar de que este «no se agota ni está asociado únicamente a lo físico-espacial» (Carrión Mena, Espacio público 80). En efecto, la materialidad del espacio físico urbano opera como soporte de un entramado de sentidos y significados que dan cuenta de las concepciones culturales dominantes de una sociedad, en un momento determinado (Nieves Rico y Segovia 42). De esta manera, las ciudades, en tanto expresiones de la cultura, pueden ser consideradas como verdaderos textos susceptibles de ser leídos e interrogados (Mangulis 87; Stachel en Sánchez Costa 6). De acuerdo con Marcús, «podemos leer la ciudad como si fuera un texto e interpretar las huellas de su construcción histórica y social

Feminismo/s 33, junio 2019, pp. 249-271 
en las calles, en las plazas, en los edificios, etc.» (19). En otras palabras, es posible hacer investigación y «(...) hacer historia con los monumentos y con las calles, las que se proponen y las que se censuran, las que se borran y las que se agregan» (Barela 5).

En línea con estas perspectivas que proponen rescatar e indagar la dimensión simbólica de las ciudades, este trabajo busca aproximarse a los modos y grados en que las nociones dominantes acerca de lo femenino encuentran acogida en la dimensión simbólica del espacio público, tomando como «huellas» de este fenómeno (es decir, como indicadores empíricos) los nombres de los caminos (avenidas, calles, senderos, etc.) de la Ciudad de Buenos Aires. En primer lugar, este estudio argumenta que la nomenclatura urbana constituye una entrada privilegiada para el análisis de esta temática, en tanto refleja y condensa una trama de sentidos y significados, memorias y visiones canónicas de la historia que, imbuidas de nociones dominantes sobre los géneros, pretenden ser legitimadas y transmitidas por este medio y que se concretan a partir de la elección de determinados nombres y la omisión de otros. Aunque en apariencia las denominaciones de los caminos pueden parecer heterogéneas e inconexas, un análisis metódico de las mismas deja al descubierto marcadas regularidades en lo referido a las poblaciones, personalidades y eventos históricos que son considerados dignos de ser recordados y aquellos que, contrariamente, son sistemáticamente silenciados. En segundo lugar, el análisis de este fenómeno permite comenzar a explorar si el aumento que se verifica en el acceso y en la participación efectiva de las mujeres en el espacio público encuentra, en alguna medida, traducción en la dimensión simbólica de la ciudad. De esta manera, este trabajo busca proponer líneas de análisis que permitan indagar la persistencia de asimetrías de género en lo que refiere a la representación de las mujeres y de otras identidades no binarias en esta dimensión de la vida social.

El presente artículo se organiza en seis secciones. En la sección dos se exponen las principales líneas de investigación con las que dialoga este trabajo para luego, en la sección tres, adentrarse en los conceptos teóricos que permiten enmarcar y analizar el fenómeno indagado. En el apartado cuatro se detalla la estrategia metodológica utilizada para la producción de los datos estadísticos sobre los caminos de la Ciudad de Buenos Aires que aquí se exponen. Luego, en la sección cinco, se presentan los principales hallazgos

Feminismo/s 33, junio 2019, pp. 249-271 
del estudio y se sugieren líneas de interpretación de los datos expuestos. Finalmente, en el apartado seis se presentan las principales conclusiones que se desprenden de este trabajo.

Este estudio no pretende ofrecer un análisis completamente acabado del fenómeno indagado sino más bien sugerir posibles líneas de exploración y análisis que abonen al estudio de la dimensión simbólica de las ciudades desde una perspectiva de género. En esta línea, se intenta dar cuenta de la importancia de la incorporación del género como variable ineludible, si lo que se busca es conocer cómo, por y para quién está siendo pensado el espacio público.

\section{HACIA UNA PROBLEMATIZACIÓN DE LAS VINCULACIONES ENTRE GÉNERO, CIUDAD Y ESPACIO PÚBLICO}

El campo de estudio sobre los géneros y las sexualidades comenzó a configurarse en la efervescente década del sesenta del siglo XX, momento en que las preocupaciones hasta entonces aisladas sobre estas temáticas se convirtieron en esfuerzos sistemáticos por construir y legitimar un campo específico de conocimiento (Palermo 40). Así, en países altamente industrializados (tal como Francia e Inglaterra) surgieron de la mano de la segunda ola del movimiento feminista los Estudios de las Mujeres (Berllucci 27). Debido a fuertes resistencias por parte de las academias más tradicionales, en sus comienzos, éstos lograron ser admitidos en las casas de estudio bajo el formato de seminarios específicos dentro de los currículos (Berllucci 31). Las autoras pioneras de este campo de estudio centraron sus esfuerzos en problematizar el saber científico tradicional denunciando que, como consecuencia de su marcado sesgo androcéntrico, había omitido, invisibilizado y excluido a las mujeres de sus análisis y producciones científicas e históricas (Berllucci 28; Maffía 1-2). Así, alrededor de la década del ochenta del siglo pasado, los Estudios de las Mujeres dieron paso a los denominados Estudios de Género. Por un lado, este corrimiento de un concepto «lego» (mujeres) a uno teórico (género) respondió en gran medida a la búsqueda de legitimarse dentro del universo de la ciencia (Burin 5-7). Por el otro, se debió a que este campo de estudio comenzaba a perfilarse como una corriente interdisciplinaria preocupada por las relaciones de poder entabladas intra e inter géneros, así como por las desigualdades que de ellas derivan en contextos socio-históricos específicos.

Feminismo/s 33, junio 2019, pp. 249-271 
Mientras las perspectivas de género tuvieron un acogimiento relativamente temprano en las humanidades y las ciencias sociales (entre las que se destacan la historia, la antropología, la sociología, las letras y la psicología), su incorporación en las disciplinas dedicadas a estudiar y diseñar el espacio y las ciudades fue relativamente tardía. En el caso de la geografía y el urbanismo, no fue hasta entrada la década del ochenta del siglo XX que las vinculaciones entre género y espacios (públicos y privados) comenzaron a estudiarse (Falú 13; Sabaté Martínez, Rodríguez Moya y Díaz Muñoz 29). Las primeras investigaciones que adoptaron una perspectiva de género para estudiar las relaciones entre las personas y las ciudades que éstas habitan, demostraron que el espacio no es neutro en términos de género (Nieves Rico y Segovia 41; Velázquez Valoria 1). Estos estudios evidenciaron que «las relaciones de género imperantes en una sociedad influyen en la configuración de los espacios» (Azcárate 2). Asimismo, advirtieron sobre la existencia de vinculaciones entre las posiciones que mujeres y varones ocupan en la sociedad y los modos en que cada uno de ellos habita, transita y percibe los espacios (Azcárate 78-79).

En Argentina, los estudios que se han preocupado por indagar las conexiones entre género y espacio público han sido escasos. Tras una exhaustiva revisión de la producción nacional sobre esta temática, Boy reconoce tres principales líneas de investigación (153-155). Este autor identifica un primer grupo de trabajos en el campo de la sociología, con los estudios realizados por Meccia y por Rapisardi y Modarelli dedicados fundamentalmente a indagar los espacios de sociabilidad homosexual, y los códigos de seducción desplegados en el espacio público de la Ciudad de Buenos Aires, en distintos momentos históricos (Boy 155-157). Según Boy, la producción académica sobre género, sexualidades y ciudades ha sido más fructífera en el campo de los estudios urbanos. Entre éstos, el autor menciona, por un lado, las investigaciones elaboradas por Carman y por Sabsay sobre los modos complejos en que el Estado busca regular (algunas veces legalmente y otras mediante el ejercicio de la violencia) el uso y apropiación territorial de la Ciudad de Buenos Aires por parte de personas con identidades de género no heteronormadas (157159). Por otro lado, Boy destaca los trabajos realizados por Ana Falú sobre las formas en las que el miedo a ser blanco de violencias en el espacio público limita el uso, disfrute y apropiación de la ciudad por parte de las mujeres,

Feminismo/s 33, junio 2019, pp. 249-271 
al mismo tiempo que condiciona su pleno ejercicio del derecho a la ciudad (159).

Por su parte, la nomenclatura urbana de la Ciudad de Buenos Aires ha sido escasamente interrogada desde una perspectiva de género. El antecedente más directo lo constituye el trabajo de investigación histórica realizado por Leticia Maronese a fines de la década del noventa del siglo pasado. Su esfuerzo quedó cristalizado en el libro Mujeres y Calles. Nomenclatura Porteña en el que esta historiadora recupera la biografía de las pocas mujeres concretas que, en ese momento, eran homenajeadas con caminos que llevaban sus nombres.

El presente trabajo retoma la preocupación de Maronese por indagar la nomenclatura urbana desde una perspectiva de género, y por explorar la presencia-ausencia de las mujeres en esta dimensión del espacio público. En línea con este propósito, a continuación se presenta el marco conceptual que servirá para iluminar los hallazgos de este estudio.

\section{EL ESPACIO PÚBLICO COMO ESFERA DE DOMINIO MASCULINO}

A lo largo de la historia, «mujeres y hombres han tenido diferentes destinos espaciales» (Nieves Rico y Segovia 43). Sin embargo, fue con el advenimiento del orden industrial moderno que (abonando a la consolidación y al funcionamiento del sistema capitalista) quedó instituida una cadena de significantes dicotómicos, entre los que se destacan: masculino/femenino, público/privado, trabajo/cuidados, razón/emoción, individuo/comunidad, economía/familia, independencia/dependencia, competencia/sacrificio, entre otras polaridades (Fraser y Gordon 116-117; Muxí Martínez y Cireddu 39; Sabaté Martínez, Rodríguez Moya y Díaz Muñoz 45-46). Puntualmente, la oposición espacio público - espacio privado contribuyó a que quedara consolidada la división sexual del trabajo, lo que significó un profundo trastocamiento para los géneros y sus dominios de acción (Collin 232-234; Sabaté Martínez, Rodríguez Moya y Díaz Muñoz 46). De esta manera, al dictado de la Carta de Atenas, se establecieron relaciones biunívocas entre espacios y funciones (Gutiérrez Mozo 10). Así, como parte de este proceso, quedaron constituidas dos esferas de acción que, hasta la actualidad, implican a los géneros en distintos modos y grados. Por un lado, el espacio público fue definido como aquel en el cual los varones deberían llevar adelante labores remuneradas de índole productiva. 
Por el otro, quedaba constituido el espacio privado, entendido como aquel en el que las mujeres serían las principales (sino únicas) responsables de las tareas reproductivas, domésticas y de cuidado, no remuneradas (Azcárate 79-81; Sabaté Martínez, Rodríguez Moya y Díaz Muñoz 45-46).

El hecho de que desde los albores de la modernidad el espacio público se haya constituido como una esfera de dominio masculino, ha tenido diversas consecuencias diacrónicas tanto en la dimensión material de las ciudades como en la simbólica. En este sentido, en gran medida, las formas en que han sido pensadas las ciudades han respondido a visiones androcéntricas de la vida social. Esto ha implicado que las ciudades contemporáneas hayan sido diseñadas, planificadas y construidas adoptando un punto de vista que, a pesar de presentarse como «neutral»y «universal», en verdad responde a las necesidades e intereses masculinos; sin tomar prácticamente en consideración las exigencias de otros grupos poblacionales que también las habitan (Muxí Martínez y Cireddu 38). En otras palabras, la configuración urbana se ha adaptado a las necesidades y particularidades del modo de vida de un ciudadano-tipo varón, cis género, blanco, de mediana edad, independiente, con plenas capacidades de movilidad, con locomoción propia y que posee un trabajo que constituye el centro de su vida (Velázquez Valoria 4). Esta operación que implica poner al «andros» (varón dominante) en el centro de la vida social, considerándolo como la medida de todas las cosas, recibe el nombre de androcentrismo (Campagnoli 75-76; Facio y Fries 274-276). Es en este sentido que se afirma que las ciudades son androcéntricas, en la medida en que sus espacios comunes fueron (y en muchos casos continúan siendo) pensados, diseñados y desarrollados por y para ser utilizados y ocupados por aquellos varones que constituyen el centro hegemónico de la vida social.

Esta visión dominante que concibe a los varones como los legítimos usuarios del espacio público también encuentra acogida en la dimensión simbólica de las ciudades. De acuerdo con Collin, «todo lo que representa un símbolo

1. A los fines del presente trabajo, este fenómeno se ha expuesto de manera esquemática y global, destacando sus principales características. Esto no supone desconocer que hayan existido importantes diferencias en los modos en que este proceso tuvo lugar en distintos contextos nacionales e incluso regionales, ni las particularidades que éste asumió para distintas poblaciones en función de los modos en que se interseccionan distintas categorías como clase, etnia y edad.

Feminismo/s 33, junio 2019, pp. 249-271 
de pertenencia común en una arquitectura está marcado de forma masculina» (236). En las estatuas, los bustos y/o los monumentos así como en los nombres de las calles, de las plazas, de los parques y de las estaciones de subte, aún prevalece al varón como principal (sino único) sujeto de referencia. En palabras de Segovia, «los hombres han hecho suyo el espacio público dominando la naturaleza, construyendo monumentos que muestren sus hazañas de conquistadores y guerreros, descubriendo y fundando ciudades» (89-90).

Este estudio pretende comenzar a rastrear, en el caso de la Ciudad de Buenos Aires, «huellas» de un posible sesgo androcéntrico en su trama simbólica. A continuación se presenta la metodología utilizada para la construcción de datos estadísticos que se analizan en las secciones subsiguientes.

\section{METODOLOGÍA DEL ESTUDIO²}

\subsection{Características de la base de datos}

Con el propósito de explorar la presencia-ausencia de mujeres en los caminos de la Ciudad de Buenos Aires, esta investigación adoptó una estrategia metodológica cuantitativa. Para la producción de los datos estadísticos que aquí se presentan se utilizó la base de datos oficial denominada «callejero. $x l s x »^{3}$, disponible para ser descargada en la página web oficial «Buenos Aires Data» del Gobierno de la Ciudad de Buenos Aires. La misma fue consultada y descargada en el mes de noviembre de 2018. El tratamiento de los datos se realizó mediante el software de procesamiento SPSS.

La base utilizada consiste en una lista de los caminos (que comprende todas las calles, avenidas, pasajes, bulevares, calles peatonales, puentes, pasajes públicos y senderos), que recorren la superficie de la Ciudad Autónoma de Buenos Aires. Cada uno de ellos se encuentra identificado por su nombre oficial y por un código numérico de identificación único. La base también provee información adicional que, por ejemplo, permite distinguir las comunas y barrios que atraviesan cada uno de los caminos.

2. Agradezco especialmente al Licenciado Leandro García Cuerva cuyas recomendaciones y aportes en la construcción de los datos hicieron posible este trabajo.

3. <https://data.buenosaires.gob.ar/dataset/calles>.

Feminismo/s 33, junio 2019, pp. 249-271 
Androcentrismo y espacio público: análisis exploratorio sobre la subrepresentación

femenina en la nomenclatura urbana de la Ciudad Autónoma de Buenos Aires

No obstante, en la base, cada camino se encuentra dividido por tramos (también identificados con un código numérico de identificación único), los cuales se corresponden con distintas alturas de un mismo camino. Así, cada fila de la base representa un tramo particular de un camino determinado.

Por otro lado, la fuente utilizada incluye tanto los caminos que tienen nombre «oficial» como aquellos cuya nominación es «no oficial». Respecto a estos últimos casos, se tomó la decisión de no considerarlos para el análisis e incluir sólo aquellos caminos cuyos nombres estuvieran ya reconocidos por el Estado, es decir, fueran «oficiales» ${ }^{4}$.

\subsection{Procedimiento de codificación}

Debido a que, como se mencionó en el apartado anterior, en la base de datos utilizada los caminos se presentan separados en tramos (por lo que un mismo camino aparece replicado en la base tantas veces como tramos en los que se encuentra dividido), se realizó una extracción aleatoria simple de un tramo por cada uno de los caminos «oficiales». Como resultado de este procesamiento, la base quedó conformada por un único tramo por camino.

Conforme a los pasos antes señalados, el universo de caminos considerado para elaborar los resultados correspondientes a la totalidad de la Ciudad consta de dos mil ciento ochenta y un casos. El mismo está compuesto por: i)

4. Los caminos con nombres «oficiales» se identifican en la base tanto: i) porque poseen un código numérico único que los identifica como tales; o, ii) porque en el campo «observaciones» de la base se aclara que, en tanto su nombre oficial ha sido designado recientemente, aún no se les ha adjudicado un código de identificación. A los efectos de incluir estos últimos casos en el procesamiento (como pertenecientes al universo de caminos con nombre oficial), se les consignó un código de identificación numérico único, utilizando valores comprendidos en el rango de 1 al 999. Los caminos «no oficiales» pueden identificarse en la base porque la variable «código» porta el valor cero. Asimismo, se presentaron casos en los que se debió realizar el proceso inverso. La base de datos utilizada incluye algunos caminos que cuentan con un código de identificación único (como ocurre con los caminos «oficiales»), pero que en el campo «nombre oficial», se detalla que el mismo aún no posee uno. En concordancia con esto, se procedió a excluir el total de estos casos del procesamiento. Al mismo tiempo, la base contiene caminos que cambian de nombre en alguno de sus tramos, aunque comparten un mismo código de identificación único. En estos casos se tomó la decisión de incluir ambos nombres con la finalidad de que la base quedara conformada por el total de denominaciones oficiales.

Feminismo/s 33, junio 2019, pp. 249-271 
aquellos caminos cuya denominación es consignada en la base como «oficial»; y, ii) los caminos que, si bien aún no cuentan con un código de identificación único, ya poseen nombre oficial, según lo especificado en el campo «observaciones» de la base.

Sobre esta selección, se llevó a cabo la codificación de los nombres de los caminos. Los códigos utilizados para ello fueron: varón, mujer, lugar (países, provincias, localidades), histórico (eventos y artefactos históricos), naturaleza (ríos, cerros, lagos, flora y fauna), cultura/arte (obras literarias y artísticas), y otros (para los casos que no se ajustaran a ninguna de las anteriores categorías). Para garantizar la exhaustividad en la asignación de códigos, cada uno de los nombres de los caminos fue cotejado en el libro Barrios, calles y plazas de la Ciudad de Buenos Aires: origen y razón de sus nombres (2008), de la Dirección General Patrimonio e Instituto Histórico del Gobierno de la Ciudad de Buenos Aires. Esto permitió verificar con precisión a qué o quiénes se busca homenajear oficialmente en cada caso. Como parte de los criterios de codificación, sólo se contabilizaron como caminos con nombre de mujer aquellos que refieren a personalidades femeninas concretas. Es decir, calles tal como Santa Elena (que refiere a un cerro) o Amalia (que es una obra literaria), no fueron contabilizados como casos de caminos que conmemoran a mujeres.

\subsection{Procesamiento de los valores por Comuna}

Para desagregar los resultados por comuna, se procedió a generar bases parciales para cada una de ellas. De esta manera, cada base quedó conformada por los caminos que, al menos en alguno de sus tramos, comprende a la respectiva comuna. Más específicamente, la condición para que un camino se incluyera en una de las bases parciales por comuna fue que al menos uno de sus tramos o una de las manos de alguno de sus tramos atravesase la superficie de la respectiva comuna. De acuerdo a lo desarrollado en este apartado, queda explicitado que un mismo camino puede estar contabilizado en los resultados de más de una comuna si, en su trayecto, atraviesa a más de una de ellas. 
Androcentrismo y espacio público: análisis exploratorio sobre la subrepresentación

femenina en la nomenclatura urbana de la Ciudad Autónoma de Buenos Aires

\section{LAS MUJERES EN LA NOMENCLATURA DE LOS CAMINOS DE LA CIUDAD DE BUENOS AIRES}

La nomenclatura urbana de la Ciudad de Buenos Aires ha sufrido distintas transformaciones a lo largo de la historia. Como ha ocurrido en la mayoría de las ciudades de América Latina, en sus comienzos esta ciudad poseía una nomenclatura costumbrista (que surgía de los propios habitantes y refería, por ejemplo, a particularidades topográficas zonales), que luego dio paso a una de tipo conmemorativa (Carrión Mena, Centro histórico 95; Maronese 13). Puntualmente en Argentina, este pasaje se dio en el siglo XVIII, momento en que las denominaciones anteriores fueron suplantadas por los nombres de las personas que protagonizaron la resistencia a las invasiones inglesas (Piñeiro 10). Desde entonces, la nomenclatura comenzó a ser utilizada como un medio a través del cual recuperar, oficializar y legitimar visiones (en gran medida dominantes) de la historia. En otras palabras, las elites políticas encontraron en los nombres de las vías públicas vehículos para transmitir una visión canónica de la historia a generaciones actuales y venideras; una estrategia efectiva si se considera «que la ciudad y el espacio público son instancias de socialización fundamentales» (Carrión Mena, Espacio público 85).

Las tres primeras mujeres en ser reconocidas en la nomenclatura urbana de la Ciudad de Buenos Aires, gracias a la ordenanza del 27 de noviembre de 1893, fueron las heroínas de las guerras de Independencia: Juana Azurduy de Padilla, Manuela Pedraza y Policarpa Salvatierra. Asimismo, ésta también incluyó a Isabel La Católica ${ }^{5}$. Varios años más tarde, ya a comienzos del siglo

5. Los motivos por los cuales estas mujeres fueron elegidas a finales del siglo XIX para ser conmemoradas son diversos. Puntualmente, Juana Azurduy de Padilla fue homenajeada en calidad de patriota del Alto Perú que batalló en las guerras de Independencia hispanoamericanas por la emancipación del Virreinato del Río de la Plata contra el Reino de España. Asimismo, se la recuerda como la primera mujer en comandar tropas en el ejército argentino. Por su parte, Manuela Pedraza fue conmemorada como heroína de las Invasiones Inglesas que luchó en la reconquista de Buenos Aires de 1806. Por otro lado, Policarpa Salvatierra (más comúnmente conocida como «La Pola») fue conmemorada como una patriota de la independencia de nueva granada, la actual Colombia (Maronese 41-48). Finalmente, Isabel La Católica fue reina de Castilla, reina consorte de Sicilia y de Aragón. La historiografía argentina la recuerda por su apoyo a los viajes de Cristóbal Colón (Maronese 113-114).

Feminismo/s 33, junio 2019, pp. 249-271 
XX, con la ordenanza del 28 de octubre de 1904 se puso nombre a trescientas setenta y dos calles, de las cuales sólo una (Santa Rosa) ${ }^{6}$ recordaba a una mujer (Maronese 14). Desde el comienzo de ese siglo hasta el año 1995 (momento en que concluye el estudio realizado por Maronese) se fueron incluyendo, aunque en forma espaciada, veinticuatro nuevos nombres de mujeres en las calles de la ciudad. En el año 1995, fue la propia Maronese quien presentó una denuncia señalando la actitud discriminatoria en la asignación de los nombres de las calles porteñas. Finalmente, ésta resultó en la incorporación (mediante la Ordenanza N. ${ }^{\circ} 49.668$ dictada por el ex Concejo Deliberante) de catorce nombres de mujeres en las nuevas calles del barrio porteño de Puerto Madero. Para ese entonces, de alrededor de dos mil cien calles trazadas y reconocidas en la Ciudad de Buenos Aires, sólo cuarenta y tres llevaban nombres de mujeres (Maronese 7).

Conforme a los resultados obtenidos en esta investigación, este fenómeno apenas se ha modificado en los años comprendidos entre 1995 y 2018. A pesar de que la cantidad de caminos que llevan nombres de mujer ha aumentado en un $49 \%$ en el período de tiempo mencionado, la acentuada subrepresentación femenina en la toponimia urbana de la Ciudad de Buenos Aires se mantiene como una constante.

Según los datos producidos para este estudio, de los dos mil ciento ochenta y un caminos con nombre oficial trazados sobre la superficie de la Ciudad de Buenos Aires, sólo sesenta y cuatro llevan el nombre de una mujer concreta, lo que representa un 3\% del total (ver gráfico 1). Sólo presenta valores inferiores, la categoría que refiere a "cultura/arte" (1\%).

6. Fue una mística y religiosa peruana, y la primera persona en ser canonizada en América (Maronese 82). 
Gráfico 1: Caminos con nombres oficiales según elemento que se homenajea, CABA, 2018 (en \%)

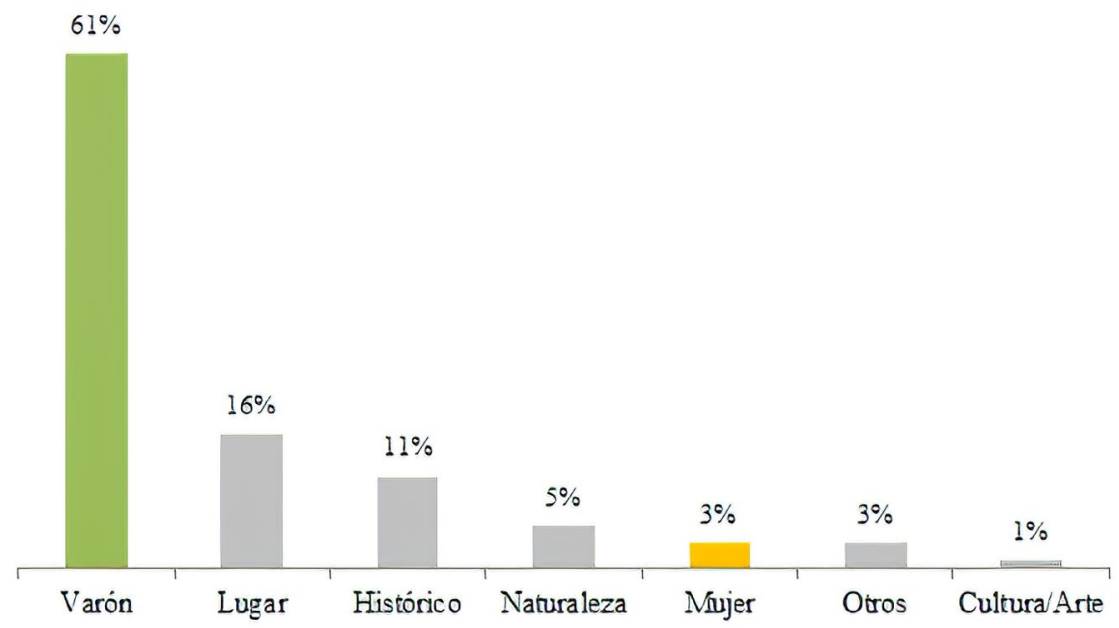

Fuente: Elaboración propia en base al callejero oficial de la Ciudad Autónoma de Buenos Aires, noviembre 2018.

Como es posible apreciar en el gráfico 1, más de la mitad (61\%) de los caminos de la Ciudad de Buenos Aires homenajean a varones concretos. De este modo, si se compara la cantidad de vías públicas que conmemoran a personas concretas, se constata que de cada veintiún caminos que en la Ciudad de Buenos Aires conmemoran a un varón, uno homenajea a una mujer. Por otro lado, todas las personalidades homenajeadas (tanto varones como mujeres) son cis género, es decir, individuos cuya identidad de género coincide con el sexo que les fue asignado al nacer. 
Androcentrismo y espacio público: análisis exploratorio sobre la subrepresentación femenina en la nomenclatura urbana de la Ciudad Autónoma de Buenos Aires

Gráfico 2: Caminos con nombres oficiales de mujer según tipo de camino, CABA, 2018 (en absolutos)

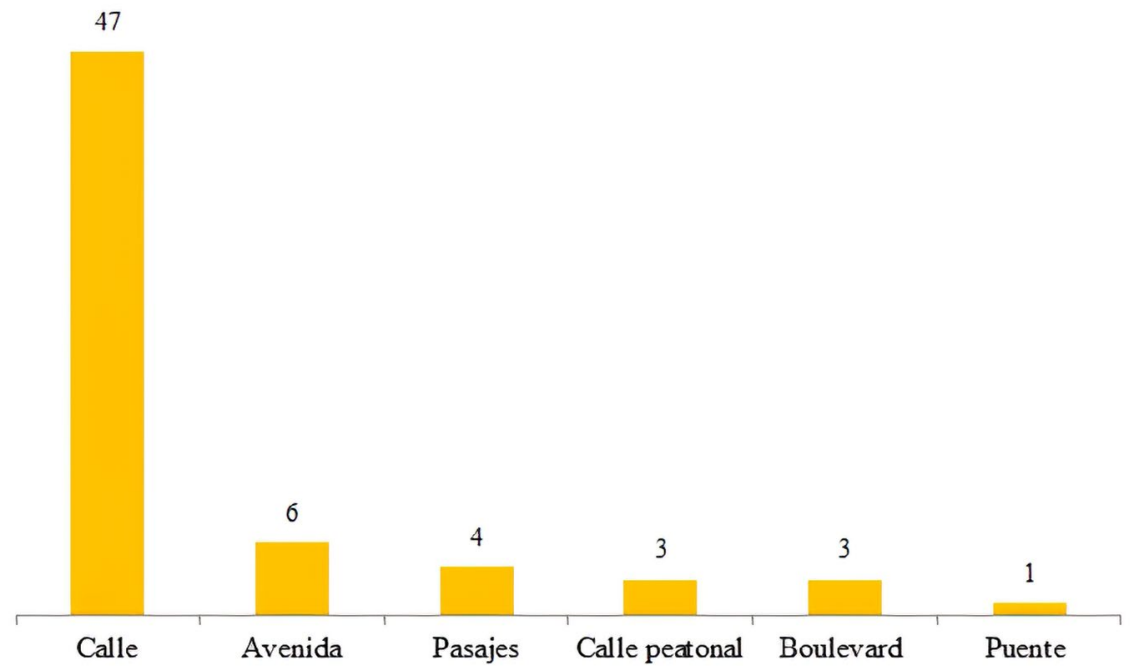

Fuente: Elaboración propia en base al callejero oficial de la Ciudad Autónoma de Buenos Aires, noviembre 2018.

Dentro del universo de vías públicas que homenajean a mujeres concretas, la «calle» (con cuarenta y siete casos) es el tipo de camino preponderante (ver gráfico 2). También, existen seis avenidas con estas características y un puente, el emblemático «Puente de La Mujer» ubicado en el barrio porteño de Puerto Madero. Sin embargo, respecto a este último, es importante señalar que esta obra (la primera realizada en América Latina por el arquitecto e ingeniero español Calatrava Vals), no busca, conforme a lo manifestado por su autor, homenajear a una personalidad concreta sino que evoca a una pareja tradicional (varón-mujer) bailando tango (Frittaoni) ${ }^{7}$.

7. Puntualmente las vías públicas con nombre de mujer según tipo de camino son calles (47): Mistral, Gabriela; Grierson, Cecilia; Janer, Ana María; del Valle, María R.; Azurduy, Juana; Pedraza, Manuela; Arenal, Concepción; Storni, Alfonsina; Pérez, Gregoria; Santa Rosa; Sánchez de Thompson, Mariquita; Pacini de Alvear, Regina; Escalada de San Martín, R.; Del Carmen; Ocampo, Victoria; Cossettini, Olga; Guevara, Trinidad; Sáenz, Manuela; de la Cruz, Sor Juana Inés; Lynch, Marta; Paine, Aime; Salotti, Martha; Eyle, 
Androcentrismo y espacio público: análisis exploratorio sobre la subrepresentación femenina en la nomenclatura urbana de la Ciudad Autónoma de Buenos Aires

Gráfico 3: Caminos con nombres oficiales de mujer por comuna, CABA, 2018 (en absolutos)

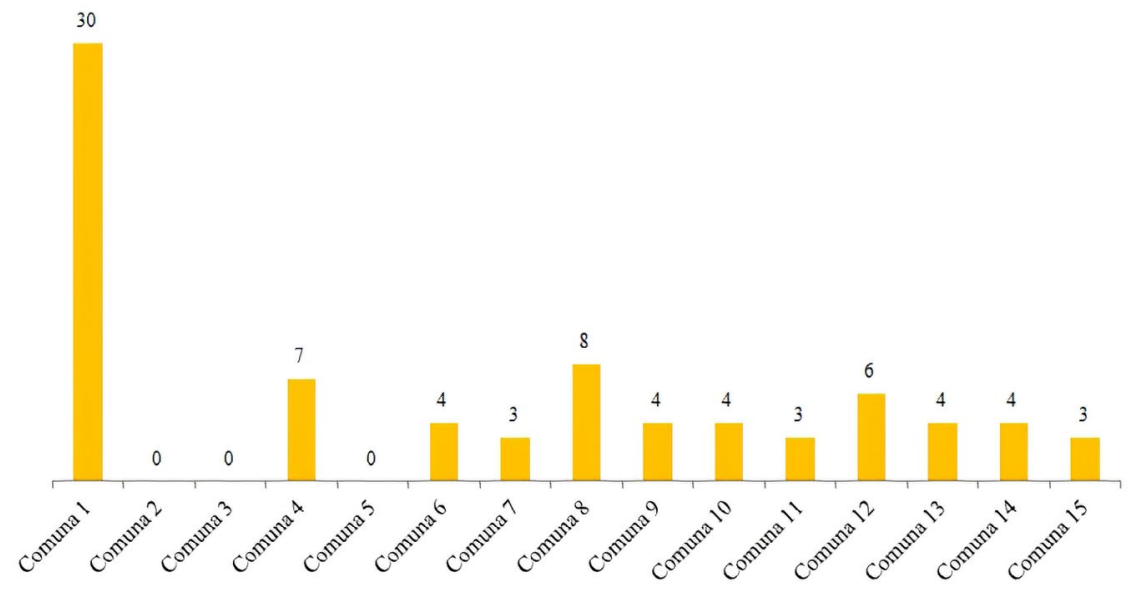

Fuente: Elaboración propia en base al callejero oficial de la Ciudad Autónoma de Buenos Aires, noviembre 2018.

En lo relativo a la distribución por comuna, los datos permitieron evidenciar que cerca de la mitad de los caminos que conmemoran a mujeres (treinta de sesenta y cuatro) se hallan dentro de los límites espaciales de la comuna 1. El hecho de que una cantidad considerable de los mismos se concentre en una única comuna (lo que se debe en gran medida a la iniciativa impulsada por Maronese en Puerto Madero antes detallada en este apartado) redunda

Petrona; Ezcurra, Encarnación; de Castro, Rosalía; Isabel La Católica; Pola; Santa Magdalena; Matorras de San Martín, Gregoria; Manso, Juana; Lanteri, Julieta; Albarracín de Sarmiento, Paula; Larroque de Roffo, Elena; Butteler; Juana de Arco; Díaz, Ana; de la Barra, Emma; Lorenzini, Carola; Santa Teresa; de la Paz y Figueroa, María Antonia; Muzilli, Carolina; Langer, Marie; Sendero Mujeres Victoriosas; Mujeres Luchadoras; Nuestra Señora de Caacupé; Marchi, María Catalina; de Ibarbourou, Juana; avenidas (6): Av. Perón, Eva; Av. Patricias Argentinas; Av. Isabel, Infanta; Av. Moreau de Justo, Alicia; Av. Rawson de Dellepiane, Elvira; Av. Curie, María; pasajes (4): Maizani, Azucena; Pagano, Angelina; O' connor, Elsa; Kavanagh, Corina; boulevard (3): Villaflor, Azucena; Guemes, Macacha; Vera Peñaloza, Rosario; calle peatonal (3): Dealessi, Pierina; Gorriti, Juana Manuela; Bemberg, María Luisa; puente (1): Puente de la Mujer.

Feminismo/s 33, junio 2019, pp. 249-271 
en una marcada segregación espacial de este tipo de referencias. Como puede observarse en el gráfico 3, los valores que registran las catorce comunas restantes son notablemente inferiores a los de la comuna 1. Incluso, las comunas 2, 3 y 5 no poseen ni un sólo camino con nombre de mujer en su territorio (ver gráfico 3).

La escasa (sino nula) presencia de mujeres concretas en la nomenclatura urbana analizada, confirma la persistencia de una profunda «ginopia de género» en los modos de nominar la ciudad. En este sentido, la miopía a lo femenino «como efecto androcéntrico de la violencia simbólica» (Campagnoli 80), redunda en la subrepresentación y/u ocultamiento de las mujeres, sus experiencias y sus aportes en los nombres que se utilizan a diario para ordenar y referenciar el espacio público. Esto pone de manifiesto uno de los tantos modos en que la cultura androcéntrica prácticamente no percibe y no nombra a las mujeres; y que de hacerlo las ubica en un lugar subsidiario o remanente (Campagnoli 79-81).

Gráfico 4: Caminos con nombres oficiales de mujer según ámbito en el que se ha destacado la conmemorada, CABA, 2018 (en absolutos)

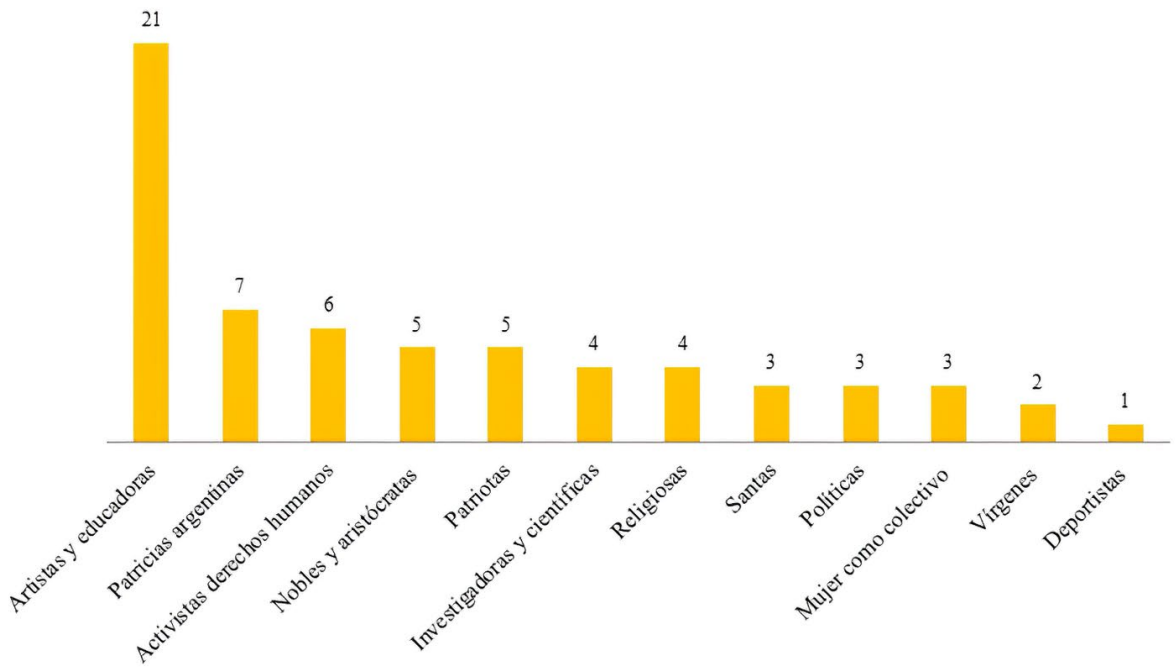

Fuente: Elaboración propia en base al callejero oficial de la Ciudad Autónoma de Buenos Aires, noviembre 2018.

Feminismo/s 33, junio 2019, pp. 249-271 
Finalmente, se analizaron los ámbitos sociales en los cuales se desempeñaron y/o destacaron las mujeres conmemoradas. Esto dejó al descubierto que la mayoría de ellas son homenajeadas por haberse desenvuelto en campos disciplinares que han sido culturalmente asociados a cualidades y/o atributos considerados como femeninos (tal como sensibilidad, paciencia, modestia, pasividad, entre otros) y que, por lo tanto, han estado históricamente feminizados. En este sentido, no resulta sorprendente que una importante cantidad de caminos conmemore a educadoras y/o artistas, considerando que en la segunda mitad del siglo XIX el magisterio era la principal (sino la única) inserción laboral considerada legítima para las mujeres y su «misión» en el proyecto educativo nacional (Anzorena 11). Al mismo tiempo, la ciudad cuenta con nueve caminos que refieren a mujeres vinculadas al ámbito religioso (santas, religiosas y vírgenes) y seis que homenajean a mujeres que se han destacado como activistas por los derechos humanos (lo que incluye personalidades que lucharon por los derechos de las mujeres y por acabar con la última dictadura militar argentina).

De esta forma, la nomenclatura urbana participa de la (re)producción, perpetuación y refuerzo de estereotipos de género a través de homenajear fundamentalmente a mujeres que se han destacado en la educación y las artes ${ }^{8}$, y prácticamente omitiendo referencias a otras personalidades femeninas que se han desempeñado con éxito en otros campos disciplinares. Tal es el caso de la política y el deporte, dos ambientes históricamente masculinizados que se han asociado al desempeño de cualidades y habilidades culturalmente atribuidas a los varones (racionalidad, fuerza física, frialdad) y que constituyen los ámbitos profesionales a los que pertenecen la menor cantidad de homenajeadas (tres y uno respectivamente). Considerando lo anteriormente señalado, podría sostenerse que las representaciones dominantes de lo que

8. Estas disciplinas si bien, como sostiene Pollock (Visión 19-50) respecto a las artes, han estado desde sus inicios codificadas como espacios eminentemente masculinos, al mismo tiempo han sido socialmente asociadas a cualidades culturalmente consideradas como femeninas (tal como la sensibilidad, la belleza, las emociones, etc.). Aunque en el campo del arte el acceso de las mujeres aún encuentra obstáculos para alcanzar la paridad de género, la educación (fundamentalmente inicial y secundaria) ha atravesado un proceso de feminización tanto en términos cuantitativos como cualitativos (con especial énfasis en áreas curriculares vinculadas a las ciencias humanas y sociales) (Anzorena 3-8). 
una determinada cultura entiende (en un momento específico) por masculino y/o femenino encuentra expresión (y persistencia a través del tiempo) en dimensiones simbólicas de las ciudades.

En este sentido, la nomenclatura urbana acoge las concepciones dominantes y asimétricas sobre los géneros persistentes en nuestra sociedad, al mismo tiempo que contribuye a la perpetuación de esta inequidad, al invitar a las personas a reconocerse a sí mismas en estos signos y lugares. En otras palabras, los modos en que los caminos son denominados en nuestras ciudades dan cuenta de cómo se representa una sociedad, pero también de cómo se proyecta hacia adelante, produciendo efectos en las subjetividades. Como sostiene Barela:

Lo cierto es que los nombres de los lugares públicos merecedores de este homenaje, con mayor o menor justicia, pasan a ser una referencia, una identificación que con el tiempo se consustancia con la vida cotidiana, dando marco a ese sentido de pertenencia entrañable que toma cuerpo en el cruce de una esquina, en la cuadra, en el barrio, en la plaza. (5)

En esta misma línea, puede sostenerse que los modos y grados en que varones y mujeres encuentran acogida en los nombres de los caminos de la ciudad «no representan de un modo más o menos fiel la realidad, sino que toma parte activa en su construcción» (Vega 224). En otras palabras, las maneras en que los géneros son representados en la toponimia urbana no constituyen meras expresiones de causas externas a ellas mismas (como pueden ser el patriarcado, el sexismo, la discriminación, etc.) sino que desempeñan un papel activo en la (re)producción de estas categorías (Pollock, Mujeres 85-90). Así, la nomenclatura urbana (con su marcada subrepresentación de las mujeres en los nombres de las vías públicas), escenifica al mismo tiempo que contribuye a perpetuar el carácter inequitativo que ha caracterizado a las relaciones entre los géneros.

\section{CONCLUSIONES}

Aunque a simple vista los nombres de los caminos de la Ciudad Autónoma de Buenos Aires puedan parecer heterogéneos e inconexos, el análisis exhaustivo de la toponimia urbana aquí presentado evidencia que existen marcadas regularidades en lo referido a las características de las poblaciones consideradas 
dignas de ser conmemoradas y de aquellas que han sido sistemáticamente invisibilizadas a lo largo de la historia. En este sentido, el género parece funcionar como un eje ordenador a partir del cual se trazan legitimidades e ilegitimidades vinculadas al acceso y a la representación en esta dimensión simbólica de la ciudad. Como ha demostrado este estudio en el caso de la Ciudad de Buenos Aires, en los nombres de los caminos que se transitan a diario aún persisten huellas cuantitativas y cualitativas del androcentrismo que ha caracterizado a la planificación y al desarrollo urbano de las ciudades modernas. Si bien la presencia de mujeres concretas en la toponimia urbana de esta ciudad ha aumentado un $49 \%$ durante el período comprendido entre 1995 y 2018 (momento en que la Ciudad de Buenos Aires pasa de tener cuarenta y tres caminos de este tipo a contar con sesenta y cuatro de ellos), la subrepresentación femenina se mantiene como una constante. Más aún, considerando que sólo el 3\% de los caminos de esta ciudad lleva en la actualidad nombre de mujeres concretas, y que más de la mitad de éstos (61\%) homenajean a varones, la equidad en este aspecto aún se encuentra muy lejos de ser alcanzada. Al mismo tiempo, la marcada concentración espacial de este tipo de referencias femeninas en apenas dos comunas de la ciudad (las comunas 1 y 8 acogen el 47,5\% de los caminos con nombre de mujer dentro de sus límites geográficos) constituye un indicador del carácter casi excepcional que hasta nuestros días adquieren los nombres femeninos dentro de la toponimia urbana.

Así, los datos estadísticos presentados en este trabajo evidencian la persistencia de una profunda ginopia de género en la nomenclatura de la Ciudad de Buenos Aires, es decir, de una miopía a lo femenino y a otras identidades no heteronormadas, que subsiste a través de los años como contracara de la prevaleciente masculinización de esta dimensión simbólica del espacio urbano. Esta ceguera de género se enfatiza al acoger dentro de la toponimia urbana principalmente a personalidades femeninas que se han destacado en campos disciplinares históricamente feminizados, así como en ocupaciones cuyo ejercicio demanda de atributos y/o cualidades que han sido socialmente asignadas y vinculadas a lo femenino. Al mismo tiempo, esto redunda en la invisibilización y/u ocultamiento de los significativos aportes que las mujeres han realizado en aquellas esferas de la vida social que han sido (y en muchos casos continúan siendo) consideradas de dominio masculino. De esta manera

Feminismo/s 33, junio 2019, pp. 249-271 
los nombres de los caminos, imbuidos de concepciones acerca de los géneros, contribuyen a reificar, legitimar y (re)actualizar estereotipos acerca de lo femenino y lo masculino, así como sobre las cualidades y aptitudes consideradas valiosas para cada uno de los géneros. Esto se traduce en una hipervisibilidad de los aportes realizados por varones a la sociedad y en una subvaloración de aquellos hechos por las mujeres.

Así, la escasez de referencias femeninas que ha caracterizado a la nomenclatura urbana de la Ciudad de Buenos Aires desde su creación hasta nuestros días, invita a pensar si esta subrepresentación de mujeres en los nombres de los caminos podría ser considerada como una frontera simbólica que participa en la demarcación de legitimidades e ilegitimidades respecto al uso y apropiación del espacio público. En este sentido, este fenómeno nos insta a seguir indagando su posible vinculación con proyectos de ciudad androcéntricos, que en gran medida han resultado excluyentes para las mujeres y otras identidades no heteronormadas. De esta manera, esta problemática instala la pregunta acerca de si es posible que las mujeres disfruten plenamente de una ciudad, cuya nomenclatura urbana prácticamente no las incluye y que, de hacerlo, las circunscriben a contextos particulares casi excepcionales, como ocurre en el barrio porteño de Puerto Madero.

El desafío de construir ciudades más inclusivas y democráticas no puede obviar la participación activa de las mujeres y de otras identidades género-sexuales en su trama material y simbólica. Para que las mujeres puedan gozar de aquello que Lefebvre denominó como el «Derecho a la Ciudad» (es decir, el derecho de los habitantes urbanos a construir, decidir y crear ciudad) es necesario que éstas participen de manera activa en la creación de las ciudades que habitan, incluida su nomenclatura, sus monumentos y sus construcciones (Molano Camargo 4). Esto, sin lugar a dudas, supondría una mayor presencia simbólica de las mujeres en la toponimia urbana así como en otros espacios públicos de la ciudad, para dejar de ocupar espacios que otros han destinado para ellas, o a pesar de ellas.

Este trabajo se propuso ofrecer una primera aproximación al estudio de la dimensión simbólica de las ciudades desde una perspectiva de género. Las líneas de análisis aquí propuestas deberán ser profundizadas y ampliadas para comprender las formas complejas en que los géneros (y entre ellos fundamentalmente las mujeres) encuentran (o no) acogida en la nomenclatura

Feminismo/s 33, junio 2019, pp. 249-271 
urbana, así como las maneras en que esta se imbrica en proyectos de ciudad que hasta el momento parecen haberse caracterizado por su marcado sesgo androcéntrico.

\section{REFERENCIAS BIBLIOGRÁFICAS}

Anzorena, Claudia. «La participación de las mujeres en el proceso de formación del Estado Nacional en Argentina de finales del siglo XIX. Reflexiones desde una perspectiva de género». Revista Iberoamericana de Educación 45.2 (2008): $1-13$.

Azcárate, Teresa. «Mujeres buscando escenas y espacios propios». Nueva Sociedad 135 (1995): 78-91.

Barela, Liniana. «Prólogo». Barrios, calles y plazas de la Ciudad de Buenos Aires: origen y razón de sus nombres. Buenos Aires: Dirección General Patrimonio e Instituto Histórico, 2008. 5.

Berllucci, Mabel. «De los estudios de la mujer a los estudios de género: han recorrido un largo camino». Las mujeres en la imaginación colectiva. Una historia de discriminación y resistencias. Comp. Ana María Fernández. Buenos Aires: Paidós, 1992. 27-51.

Boy, Martín. «El otro espacio público en los estudios urbanos de la Argentina actual: el género y las sexualidades también construyen ciudad». Quid 16: Revista del Área de Estudios Urbanos 9 (2018): 153-167.

Burin, Mabel. «Estudios de Género. Reseña histórica». Género y Familia. Poder, amor y sexualidad en la construcción de la subjetividad. Eds. Mabel Burin e Irene Meler. Buenos Aires: Editorial Paidós, 2010. 19-30.

Campagnoli, Mabel. «¡Anda a lavar los platos!». Para una didáctica con perspectiva de género. Buenos Aires: Miño y Dávila, 2015. 59-104.

Carrión Mena, Fernando. «Centro histórico: la polisemia del espacio público». Centro-h, Revista de la Organización Latinoamericana y del Caribe de Centros Históricos 2 (2008): 89-96.

—.»Espacio público: punto de partida para la alteridad». Espacios públicos y construcción social. Hacia un ejercicio de ciudadanía. Ed. Olga Segovia. Santiago de Chile: Ediciones SUR, 2007. 79-97.

Collin, Françoise. «Espacio doméstico, espacio público». Ciudad y mujer. Madrid: Seminario permanente Mujer y Ciudad, 1994. 231-237.

Facio, Alda y Lorena Fries. «Feminismo, género y patriarcado». Academia: revista sobre enseñanza del derecho de Buenos Aires 3 (2005): 259-294. 
Falú, Ana María. «El derecho de las mujeres a la ciudad. Espacios públicos sin discriminaciones y violencias». Revista Vivienda y Ciudad 1 (2014): 10-28.

Fraser, Nancy y Linda Gordon. «Genealogía del término dependencia». Fortunas del feminismo. Ed. Nancy Fraser. Madrid: Traficantes de sueños, 2015. 109-138.

Frittaoni, Verónica. «Aniversario en Puerto Madero. La historia del Puente de la Mujer, un ícono porteño que cumple 15 años». Diario Clarín. Sección Ciudades 24 noviembre 2016.

Gutiérrez Mozo, María Elia. «Introducción a la arquitectura y el urbanismo con perspectiva de género». Feminismo/s 17 (2011): 9-22.

Maffia, Diana. «Epistemología feminista: La subversión semiótica de las mujeres en la ciencia». Revista Venezolana de Estudios de la Mujer 12.28 (2007): 63-98.

Mangulis, Mario. Sociología de la cultura. Conceptos y problemas. Buenos Aires: Editorial Biblos, 2009.

Marcús, Juliana. «Introducción. La ciudad en disputa». Ciudad Viva. Disputas por la producción sociocultural del espacio urbano en la Ciudad de Buenos Aires. Comp. Juliana Marcús. Buenos Aires: Teseo, 2017. 17-32.

Maronese, Leticia. Mujeres y calles. Nomenclatura porteña. Buenos Aires: Concejo Deliberante de la Ciudad de Buenos Aires, 1997.

Molano Camargo, Frank. «El derecho a la ciudad: de Henri Lefebvre a los análisis sobre la ciudad capitalista contemporánea». Folios 44 (2016): 3-19.

Muxí Martínez, Zaida y Alessandra Cireddu. «Urbanismo desde una perspectiva de género». Ciudades Vivibles, Segundo Congreso Iberoamericano de Jóvenes Comprometidos con las Ciudades Guadalajara,México. 19 Mar. 2014.

Nieves Rico, María y Olga Segovia. ¿Quién cuida en la Ciudad? Aportes para políticas urbanas de igualdad. Vol. 150. Santiago: Libros de la Comisión Económica para América Latina y el Caribe (CEPAL), 2007.

Palermo, Alicia Itatí. «El origen de los estudios de educación y género en las universidades argentinas». Mujeres y universidad en España y América Latina. Eds. Consuelo Flecha García y Alicia Itatí Palermo. Buenos Aires: Miño y Dávila, 2008. 39-55.

Piñeiro, Alberto Gabriel. La nomenclatura de Buenos Aires: su historia y su historiografía. Buenos Aires: Dirección General Patrimonio e Instituto Histórico, 2018.

Pollock, Griselda. «Mujeres ausentes (un replanteamiento de antiguas reflexiones sobre imágenes de la mujer». Revista de Occidente 127 (1991): 85-90. 
-.Visión y diferencia. Feminismo, feminidad e historias del arte. Buenos Aires: Fiordo, 2013.

Sabaté Martínez, Ana, Juana Rodríguez Moya y María de los Ángeles Díaz Muñoz. Mujeres, espacio y sociedad. Hacia una geografía del género. Madrid: Síntesis, 1995.

Sánchez Costa, Fernando. «Los mapas de la memoria. Nombres de calles y políticas de memoria en Barcelona y Madrid». Revista de Historia Contemporánea 9 (2009): 1-29.

Segovia Marín, Olga. «Espacio y género». Proposiciones 21 (1992):89-95.

Vega, Elo. «¿Una violencia invisible? Las mujeres en los monumentos públicos». Boletín de Arte, Departamento de Historia del Arte, Universidad de Málaga 37 (2016): 213-225.

Velázquez Valoria, Isabel. «El tiempo de las cerezas. Reflexiones sobre la ciudad desde el feminismo». Cuadernos de investigación urbanística 42 (2004): 67-74. 